PROCEEDINGS OF THE

AMERICAN MATHEMATICAL SOCIETY

Volume 125, Number 2, February 1997, Pages 455-461

S 0002-9939(97)03570-3

\title{
RADON TRANSFORM ON SPACES OF CONSTANT CURVATURE
}

\author{
CARLOS A. BERENSTEIN, ENRICO CASADIO TARABUSI, AND ÁRPÁD KURUSA \\ (Communicated by Peter Li)
}

\begin{abstract}
A correspondence among the totally geodesic Radon transformsas well as among their duals - on the constant curvature spaces is established, and is used here to obtain various range characterizations.
\end{abstract}

\section{INTRODUCTION}

The totally geodesic Radon transform on spaces of constant curvature has been widely studied (see [Hg1], [Hg2], [BC1], [Ku1], to quote only a few). Yet many of the known results, in spite of their similarities, were obtained on each such space independently. The idea of relating these transforms by projecting each space to the Euclidean one appeared independently in [BC2] (for negative curvature) and [Ku4] (for curvature of any sign), and was used to obtain, respectively, range characterizations of the Radon transform on the hyperbolic space and support theorems on all constant curvature spaces.

In this paper we extend and exploit further this correspondence, establishing an explicit formula relating the dual Radon transforms on the different constant curvature spaces, and proving various range characterizations for the Radon transform and its dual on the Euclidean and elliptic space.

The third author wishes to thank the Soros Foundation and MIT for supporting his visit at MIT, during which this paper was conceived.

\section{Preliminaries}

Let $\mathbf{M}_{\kappa}^{n}$ be an $n$-dimensional simply connected complete Riemannian manifold of constant curvature $\kappa$. Normalizing the metric so that $\kappa$ equals $-1,0$, or +1 , we get, respectively, the hyperbolic space $\mathbf{H}^{n}$, the Euclidean space $\mathbf{R}^{n}$, and the sphere $\mathbf{S}^{n}$-or its two-to-one quotient, the projective space $\mathbf{P}^{n}$. (The subscripts $+1,-1$ will henceforth be replaced by,+- , respectively.)

Received by the editors August 8, 1995.

1991 Mathematics Subject Classification. Primary 44A12; Secondary 53C65, 51M10.

Key words and phrases. Radon transform, spaces of constant curvature, totally geodesic submanifolds, spherical harmonics, moment conditions.

The first author was partially supported by NSF grants DMS9225043 and EEC9402384.

This research was in part accomplished during the second author's stay at the University of Maryland, whose hospitality is hereby acknowledged.

The third author was partially supported by the Hungarian NSF grants T4427, F016226, W075452, and T020066. 
For a fixed $k$, with $1 \leq k<n$, let $\xi$ be an arbitrary $k$-dimensional totally geodesic submanifold of $\mathbf{M}_{\kappa}^{n}$. The $k$-dimensional Radon transform $R_{\kappa} f$ (the indication of $n, k$ will be omitted) of a function $f$ on $\mathbf{M}_{\kappa}^{n}$ which is integrable on each $\xi$ is given by

$$
R_{\kappa} f(\xi)=\int_{\xi} f(x) d x,
$$

where $d x$ is the volume element on $\xi$ induced by the metric of $\mathbf{M}_{\kappa}^{n}$. In $\mathbf{S}^{n}$, whenever $x \in \xi$ then also $-x \in \xi$, so that $R_{+}$cannot separate antipodal points, i.e., it vanishes on odd functions: for this reason we will only consider even functions on $\mathbf{S}^{n}$, or, equivalently (except for a factor 2 in the integrals), will take $\mathbf{M}_{+}^{n}$ to be $\mathbf{P}^{n}$.

Let $\Gamma_{\kappa}^{n, k}$ be the set of all $k$-dimensional totally geodesic submanifolds of $\mathbf{M}_{\kappa}^{n}$, equipped with the natural differentiable structure: if $\kappa=0$ it is the usual affine Grassmannian $G^{n, k}$ of $k$-planes. The $k$-dimensional dual Radon transform $R_{\kappa}^{*} \phi$ of, say, a continuous function $\phi$ on $\Gamma_{\kappa}^{n, k}$ is defined as

$$
R_{\kappa}^{*} \phi(x)=\int_{\xi \ni x} \phi(\xi) d \xi,
$$

where $x \in \mathbf{M}_{\kappa}^{n}$ and $d \xi$ is the measure on the subset $\left\{\xi \in \Gamma_{\kappa}^{n, k}: \xi \ni x\right\}$ induced by the isotropy group of $x$ in the automorphism group of $\mathbf{M}_{\kappa}^{n}$, normalized as in $[\mathrm{Hg} 1]$.

The Riemannian metric of $\mathbf{M}_{\kappa}^{n}$ is determined by the size function $\nu_{\kappa}$, defined on $[0, \pi / 2)$ for $\kappa=+1$, and on $[0,+\infty)$ for $\kappa=-1,0$, that gives the radius $\nu_{\kappa}(r)$ of the Euclidean sphere isometric to the geodesic sphere of radius $r$ in $\mathbf{M}_{\kappa}^{n}$. Taking geodesic polar coordinates in $\mathbf{M}_{\kappa}^{n}$ with respect to a fixed reference point $o$, the function

$$
\mu_{\kappa}=\nu_{\kappa} /\left(d \nu_{\kappa} / d r\right)
$$

generates the map $p_{\kappa}: \mathbf{M}_{\kappa}^{n} \rightarrow \mathbf{R}^{n}$ given by

$$
p_{\kappa}\left(\operatorname{Exp}_{o} r \omega\right)=\mu_{\kappa}(r) \omega,
$$

where $\omega$ is a unit vector in the tangent space $T_{o} \mathbf{M}_{\kappa}^{n}$, identified with $\mathbf{R}^{n}$. (Actually $p_{+}$is defined on the complement in $\mathbf{P}^{n}$ of the projective hyperplane, henceforth called the distinguished equator $e_{o}$, orthogonal to $o$. The $\mathbf{S}^{n}$ version of $p_{+}$is of course the composition with the canonical quotient map.) The following table gives $\nu_{\kappa}$, $\mu_{\kappa}$ explicitly.

\begin{tabular}{|c||c|c|c|}
\hline $\mathbf{M}_{\kappa}^{n}$ & $\kappa$ & $\nu_{\kappa}$ & $\mu_{\kappa}$ \\
\hline \hline $\mathbf{H}^{n}$ & -1 & $\sinh r$ & $\tanh r$ \\
\hline $\mathbf{R}^{n}$ & 0 & $r$ & $r$ \\
\hline $\mathbf{S}^{n}$ or $\mathbf{P}^{n}$ & +1 & $\sin r$ & $\tan r$ \\
\hline
\end{tabular}

The map $p_{\kappa}$ has the property of taking geodesic segments to geodesic segments, although not preserving their arc length: the map $\Gamma_{\kappa}^{n, k} \rightarrow G^{n, k}$ thus induced will also be denoted by $p_{\kappa}$. (If $\kappa=+1$ this is only defined on a Zariski dense open subset of $\Gamma_{+}^{n, k}$.)

The distance of $\xi$ from $o$ in $\mathbf{M}_{\kappa}^{n}$ will be denoted by $|\xi|$; if $k=n-1$ we shall parametrize $\xi$ as $|\xi| \omega$, where $\omega \in T_{o} \mathbf{M}_{\kappa}^{n}$ is the unit direction of the closest point of $\xi$ to $o$. 


\section{Correspondence among Radon transforms AND AMONG THEIR DUALS}

The following is proved in [Ku4] and, for the hyperbolic case, in [BC2].

Theorem 3.1. Let $f \in L^{1}\left(\mathbf{R}^{n}\right)$.

(1) If $g$ is the even function on $\mathbf{S}^{n}$ (but not defined on $e_{o}$ ) given by $g\left(p_{+}^{-1}(x)\right)=$ $\left(1+|x|^{2}\right)^{(k+1) / 2} f(x)$ for all $x \in \mathbf{R}^{n}$, then

$$
R_{0} f(\xi)=\left(1+|\xi|^{2}\right)^{-1 / 2} R_{+} g\left(p_{+}^{-1}(\xi)\right) \quad \text { for every } k \text {-plane } \xi \text { in } \mathbf{R}^{n} \text {. }
$$

(2) Assume that $f$ has support in the unit ball $\mathbf{B}^{n}$ of $\mathbf{R}^{n}$ : if $g$ is the function on $\mathbf{H}^{n}$ given by $g\left(p_{-}^{-1}(x)\right)=\left(1-|x|^{2}\right)^{(k+1) / 2} f(x)$ for all $x \in \mathbf{R}^{n}$, then

$$
R_{0} f(\xi)=\left(1-|\xi|^{2}\right)^{-1 / 2} R_{-} g\left(p_{-}^{-1}(\xi)\right) \quad \text { for every } k \text {-plane } \xi \text { in } \mathbf{R}^{n} \text {. }
$$

(These multiplication operators and the Radon transforms are all positive operators, and only integrability along $k$-planes, or totally geodesic submanifolds, is actually required, so $f$ can be taken to belong to a larger function space than $L^{1}$.)

Similar relations hold for the dual Radon transform: it was proved in [BC2] for the hyperbolic case, but the same method can be used in general, as we now show.

Theorem 3.2. Let $\phi \in \mathcal{C}\left(G^{n, k}\right)$.

(1) If $\psi$ is the function on $\Gamma_{+}^{n, k}$ (not defined on the totally geodesic submanifolds contained in $\left.e_{o}\right)$ given by $\psi\left(p_{+}^{-1}(\xi)\right)=\left(1+|\xi|^{2}\right)^{n / 2} \phi(\xi)$ for every $k$-plane $\xi$ in $\mathbf{R}^{n}$, then

$$
R_{0}^{*} \phi(x)=\left(1+|x|^{2}\right)^{(k-n) / 2} R_{+}^{*} \psi\left(p_{+}^{-1}(x)\right) \quad \text { for every } x \in \mathbf{R}^{n} .
$$

(2) If $\psi$ is the function on $\Gamma_{-}^{n, k}$ given by $\psi\left(p_{-}^{-1}(\xi)\right)=\left(1-|\xi|^{2}\right)^{n / 2} \phi(\xi)$ for every $k$-plane $\xi$ in $\mathbf{R}^{n}$, then

$$
R_{0}^{*} \phi(x)=\left(1-|x|^{2}\right)^{(k-n) / 2} R_{-}^{*} \psi\left(p_{-}^{-1}(x)\right) \quad \text { for every } x \in \mathbf{B}^{n} .
$$

Proof. (1) For $\kappa=+1$ consider the functions

$$
\begin{array}{lll}
\rho\left(p_{\kappa}^{-1}(x)\right)=\left(1+\kappa|x|^{2}\right)^{(-k-1) / 2}, & \rho^{\prime}\left(p_{\kappa}^{-1}(x)\right)=\left(1+\kappa|x|^{2}\right)^{(k-n) / 2}, \\
\sigma\left(p_{\kappa}^{-1}(\xi)\right)=\left(1+\kappa|\xi|^{2}\right)^{-1 / 2}, & \sigma^{\prime}\left(p_{\kappa}^{-1}(\xi)\right)=\left(1+\kappa|\xi|^{2}\right)^{-n / 2},
\end{array}
$$

and the maps $\Phi_{\kappa}, \Phi_{\kappa}^{\prime}, \Psi_{\kappa}, \Psi_{\kappa}^{\prime}$ given by

$$
\begin{array}{ll}
\Phi_{\kappa}\left(f \circ p_{\kappa}\right)=\left(\rho \circ p_{\kappa}^{-1}\right) f, & \Phi_{\kappa}^{\prime}\left(f \circ p_{\kappa}\right)=\left(\rho^{\prime} \circ p_{\kappa}^{-1}\right) f, \\
\Psi_{\kappa}\left(\phi \circ p_{\kappa}\right)=\left(\sigma \circ p_{\kappa}^{-1}\right) \phi, & \Psi_{\kappa}^{\prime}\left(\phi \circ p_{\kappa}\right)=\left(\sigma^{\prime} \circ p_{\kappa}^{-1}\right) \phi,
\end{array}
$$

for $f \in \mathcal{D}\left(\mathbf{R}^{n}\right)$ and $\phi \in \mathcal{C}\left(G^{n, k}\right)$. The Jacobian determinant of $p_{\kappa}$ is easily seen to be $J p_{\kappa}=\left(d \nu_{\kappa} / d r\right)^{-n-1}$ (cf. [Ku4, Figure 1]). Therefore, if $f^{\prime}$ is also in $\mathcal{D}\left(\mathbf{R}^{n}\right)$ and if $g=f \circ p_{\kappa}$ and $g^{\prime}=f^{\prime} \circ p_{\kappa}$, then

$$
\left\langle\Phi_{\kappa} g, \Phi_{\kappa}^{\prime} g^{\prime}\right\rangle_{\mathbf{R}^{n}}=\int_{\mathbf{R}^{n}}\left(\rho g \rho^{\prime} g^{\prime}\right) \circ p_{\kappa}^{-1}=\int_{\mathbf{M}_{\kappa}^{n}} \rho \rho^{\prime} g g^{\prime} J p_{\kappa}=\int_{\mathbf{M}_{\kappa}^{n}} g g^{\prime}=\left\langle g, g^{\prime}\right\rangle_{\mathbf{M}_{\kappa}^{n}},
$$

because $1+\kappa\left|p_{\kappa}\right|^{2}=\left(d \nu_{\kappa} / d r\right)^{-2}$, hence $\rho \rho^{\prime} J p_{\kappa}=\left(d \nu_{\kappa} / d r\right)^{(k+1)+(n-k)+(-n-1)}=1$. Since $\Phi_{\kappa}$ is a topological isomorphism of $\mathcal{D}\left(\mathbf{S}^{n}, e_{o}\right)$, the space of even functions on $\mathbf{S}^{n}$ vanishing on a neighborhood of $e_{o}$, with $\mathcal{D}\left(\mathbf{R}^{n}\right)$, it follows that the identity $\left\langle\Phi_{\kappa} g, \Phi_{\kappa}^{\prime} g^{\prime}\right\rangle_{\mathbf{R}^{n}}=\left\langle g, g^{\prime}\right\rangle_{\mathbf{M}_{\kappa}^{n}}$ implies $\Phi_{\kappa}^{\prime}=\left(\Phi_{\kappa}^{*}\right)^{-1}$. Similarly we get $\Psi_{\kappa}^{\prime}=\left(\Psi_{\kappa}^{*}\right)^{-1}$. 
With this notation Theorem 3.1 reads $R_{0}=\Psi_{\kappa} R_{\kappa} \Phi_{\kappa}^{-1}$; taking adjoints we obtain $R_{0}^{*}=\left(\Phi_{\kappa}^{-1}\right)^{*} R_{\kappa}^{*} \Psi_{\kappa}^{*}=\Phi_{\kappa}^{\prime} R_{\kappa}\left(\Psi_{\kappa}^{\prime}\right)^{-1}$.

(2) The argument for $\kappa=-1$ is verbatim the same, except that $f, f^{\prime}$ must have support in $\mathbf{B}^{n}$ (the expression of $J p_{-}$was found in [BC2]), and $\Phi_{-}$is a topological isomorphism of $\mathcal{D}\left(\mathbf{H}^{n}\right)$ with its image, which is strictly contained in $\mathcal{D}\left(\mathbf{R}^{n}\right)$.

Observe that $\Phi_{+}$is also a topological isomorphism of $\mathcal{S}\left(\mathbf{S}^{n}, e_{o}\right)$, the space of even functions on $\mathbf{S}^{n}$ vanishing on $e_{o}$ to infinite order, with $\mathcal{S}\left(\mathbf{R}^{n}\right)$.

\section{Consequences}

Let us provide some applications of the results of the preceding section. Support theorems on the various constant curvature spaces were previously obtained in [Ku4], while range characterizations in the hyperbolic space were proved in [BC2]. We first state for general $k$ a result known for $k=n-1$ (cf. [Gu], [Gr], [St]): by $\mathcal{C}_{\text {even }}^{\infty}$ we shall denote the space of smooth even functions.

Theorem 4.1 ([St, Lemma 4.3]). The Radon transform $R_{+} \quad: \quad \mathcal{C}_{\text {even }}^{\infty}\left(\mathbf{S}^{n}\right) \rightarrow$ $\mathcal{C}^{\infty}\left(\Gamma_{+}^{n, k}\right)$ is an injection for general $k$, and is onto when $k=n-1$.

Proof. The spherical harmonics expansion of $f \in \mathcal{C}_{\text {even }}^{\infty}\left(\mathbf{S}^{n}\right)$ is $\sum_{m, j} f_{m, j} Y_{m, j}$, where $Y_{m, j}$ has even degree $m$, and $f_{m, j}$ tends to zero of infinite order as $m \rightarrow \infty$. It is known that the spherical harmonics 'diagonalize' the Radon transform: using the notation of [St], we have the expansion $R_{+} f=\sum_{m, j} c_{m} f_{m, j} Z_{m, 0, \ldots, 0 ; j}$ (with $\min \{k+1, n-k\}$ subscripts before the semicolon), where $R_{+} Y_{m, j}=c_{m} Z_{m, 0, \ldots, 0 ; j}$ for all $m, j$, and

$$
c_{m}=(-1)^{m} \frac{\Gamma(m+1 / 2) \Gamma((k+1) / 2)}{\sqrt{\pi} \Gamma(m+(k+1) / 2)}
$$

by [St, Lemma 4.1] - the space $\Gamma_{+}^{n, k}$ is identified with $O(n+1) / O(k+1) \times O(n-k)$. The asymptotic behavior of $c_{m}$ is that of $m^{-k / 2}$, hence also the sequence $c_{m} f_{m, j}$ tends to zero of infinite order, and we conclude that $R_{+} f \in \mathcal{C}^{\infty}\left(\Gamma_{+}^{n, k}\right)$. Note that $Y_{m, j}, Z_{m, 0, \ldots, 0 ; j}$ are not normalized, but both their norms behave asymptotically like $m^{-1 / 4}$, so normalizing would not change the behavior of $c_{m}$.

Since multiplication by the sequence $c_{m}^{-1}$ also preserves infinitesimal sequences of infinite order, the converse is analogous.

To extend [So, Theorem 7.7] we need to introduce moment conditions different from the usual ones.

Definition 4.2. We say that $\phi \in \mathcal{C}\left(G^{n, k}\right)$ satisfies the $m$-th refined moment condition if for every $\xi^{\prime} \in G^{n, k+1}$ containing 0 there exists a degree $m$ homogeneous polynomial $P_{\xi^{\prime}}$ on $\xi^{\prime}$ such that for every unit vector $\omega \in \xi^{\prime}$ the function

$$
t \mapsto t^{m} \phi\left(t \omega+\omega^{\perp} \cap \xi^{\prime}\right)
$$

is integrable on $(-\infty,+\infty)$ uniformly in $\xi^{\prime}, \omega$ and the integral equals $P_{\xi^{\prime}}(\omega)$. 
Define

$$
\begin{aligned}
& \mathcal{A}_{\ell}^{n}=\left\{f \in \mathcal{C}^{\infty}\left(\mathbf{R}^{n}\right):\right. \\
& f(x)=O\left(|x|^{-\ell}\right), \lim _{t \rightarrow \infty}(d / d t)^{j}\left(t^{\ell}\left[f\left(x_{0}+t x\right)-f\left(x_{0}-t x\right)\right]\right)=0 \\
&\text { for all } \left.x_{0}, x \in \mathbf{R}^{n} \text { and } j \geq 0\right\}, \\
& \mathcal{B}_{\ell}^{n, k}=\left\{\phi \in \mathcal{C}^{\infty}\left(G^{n, k}\right):\right. \\
& \phi(\xi)=O\left(|\xi|^{-\ell}\right), \lim _{t \rightarrow \infty}(d / d t)^{j}\left(t^{\ell}\left[\phi\left(\xi_{0}+t x\right)-\phi\left(\xi_{0}-t x\right)\right]\right)=0
\end{aligned}
$$

for all $\xi_{0} \in G^{n, k}, x \in \mathbf{R}^{n}$, and $\left.j \geq 0\right\}$.

(Note that $\mathcal{A}_{\ell}^{n}=\mathcal{B}_{\ell}^{n, 0}$, if we identify $\mathbf{R}^{n}$ with $G^{n, 0}$.) The extension of Solmon's result is as follows.

Theorem 4.3. For $1 \leq k \leq n-1$ the $k$-dimensional Radon transform $R_{0}$ is an injection of $\mathcal{A}_{k+1}^{n}$ into $\mathcal{B}_{1}^{n, k}$, and a bijection if $k=n-1$.

If $f \in \mathcal{A}_{k+1}^{n}$, then $\phi=R_{0} f$ satisfies the $m$-th refined moment condition if and only if $f=O\left(|x|^{-k-m-2}\right)$ for $|x| \rightarrow \infty$.

Proof. The first statement is obtained by pulling back Theorem 4.1 via Theorem 3.1: we omit the details.

Integrating in polar coordinates around the closest point of a $k$-plane $\xi$, one easily verifies that, in general, if $f(x)=O\left(|x|^{-k-\alpha}\right)$ then $R_{0} f(\xi)=O\left(|\xi|^{-\alpha}\right)$ for $\alpha>0$ : the "if" part follows from this and the classical Helgason moment conditions, applied to the restriction of $f, \phi$ to each $(k+1)$-plane $\xi^{\prime}$ through 0 .

Also for the "only if" part we shall consider one $\xi^{\prime}$ at a time, proving the required decay of $f$ on it, and then concluding on the whole of $\mathbf{H}^{n}$ by uniformity. Therefore we can assume that $n=k+1$ : we have to prove that if $t \mapsto$ $t^{m} R_{0} f\left(t \omega+\omega^{\perp}\right)$ is integrable on $(-\infty,+\infty)$ uniformly in $\omega \in \mathbf{S}^{n-1}$, then $f=$ $O\left(|x|^{-n-m-1}\right)$. If $g$ is the function on $\mathbf{S}^{n}$ obtained pulling back $f$ via Theorem 3.1, namely $g(r \omega)=f(\tan r \omega) / \cos ^{n} r$, then we have $g \in \mathcal{C}^{\infty}\left(\mathbf{S}^{n}\right)$, and $R_{+} g \in$ $\mathcal{C}^{\infty}\left(\Gamma_{+}^{n, n-1}\right)$; so $R_{+} g(r \omega) \sin ^{m} r \cos ^{-m-1} r$ is integrable on $[0, \pi / 2]$ uniformly in $\omega$. Since $R_{+} g(r \omega)$ is smooth at $r=\pi / 2$, from its Taylor expansion we deduce that it vanishes there of order (at least) $m+1$ uniformly in $\omega$. Therefore so does each spherical harmonic expansion coefficient $\left(R_{+} g\right)_{i, \ell}(r)$ (in $\left.\mathbf{S}^{n-1}\right)$ uniformly in $i, \ell$. By $\left[\mathrm{Ku} 2\right.$, Theorem 2.5( $\left.\left.\mathrm{rni}^{\prime}\right)\right]$ the coefficients of $g$ are

$$
\begin{aligned}
g_{i, \ell}(t)=c_{i, \ell} D \int_{t}^{\pi / 2} & \left(R_{+} g\right)_{i, \ell}(r) \\
& \cdot C_{i, \ell}^{n / 2-1}\left(\frac{\tan r}{\tan t}\right)\left(\frac{\tan ^{2} r}{\tan ^{2} t}-1\right)^{(n-3) / 2} \frac{\sin ^{n-2} t}{\sin r} \cot ^{n-2} r d r
\end{aligned}
$$

where

$$
D= \begin{cases}\frac{d}{d t} \prod_{j=1}^{n / 2-1}\left(\frac{d^{2}}{d t^{2}}+(2 j)^{2}\right) & \text { if } n \text { is even } \\ \prod_{j=1}^{(n-1) / 2}\left(\frac{d^{2}}{d t^{2}}+(2 j-1)^{2}\right) & \text { if } n \text { is odd }\end{cases}
$$


and $C_{i, \ell}^{n / 2-1}$ is the Gegenbauer polynomial. The change of variable $s=\tan r / \tan t$ and an elementary computation show that $g_{i, \ell}(r)$ vanishes at $r=\pi / 2$ of order (at least) $m+1$ uniformly in $i, \ell$; therefore so does $g(r \omega)$ uniformly in $\omega$. The result now follows from translating these vanishing conditions into the corresponding decay conditions for $f$.

Theorem 4.3 is similar to the so-called support theorems for the $k$-plane transform, and in the case $k=n-1$ it provides a less technical proof of the Schwartz theorem for the Radon transform (see [Hg1], [So]).

Corollary 4.4. If the $k$-plane transform $\phi=R_{0} f$ of $f \in \mathcal{A}_{k+1}^{n}$ satisfies the $m$-th refined moment condition for all $m$, then $f \in \mathcal{S}\left(\mathbf{R}^{n}\right)$.

The $(n-1)$-plane transform is a bijection of $\mathcal{S}\left(\mathbf{R}^{n}\right)$ with the space of $\phi \in$ $\mathcal{S}\left(G^{n, n-1}\right)$ that satisfy the $m$-th refined moment condition for all $m$.

A more accurate estimate of the coefficients of the spherical harmonics expansion in the proof of Theorem 4.1 can be used to replace $\mathcal{C}^{\infty}$-smoothness with finite order differentiability in Theorem 4.3, thereby generalizing the main theorem of [MS].

It is known (see, e.g., [Gr]) that, in the sphere case and for $k=n-1$, the Radon transform $R_{+}$can be identified with its dual $R_{+}^{*}$ by identifying great spheres with their polar point(s). In view of Theorem 4.1 we can thus state the following result, related to Hertle's [Ht].

Theorem 4.5. For $k=n-1$, the dual Radon transform $R_{0}^{*}$ is a bijection of $\mathcal{B}_{n}^{n, n-1}$ with $\mathcal{A}_{1}^{n}$.

A function $\phi \in \mathcal{B}_{n}^{n, n-1}$ satisfies the moment conditions

$$
\int_{-\infty}^{\infty} t^{j} \phi(t \omega) d t=P_{j}(\omega) \quad \text { for all } j=0, \ldots, m
$$

(where $P_{j}$ is a homogeneous polynomial of degree $j$ on $\mathbf{S}^{n-1}$ ) if and only if $f=$ $R_{0} \phi \in \mathcal{A}_{1}^{n}$ and $f=O\left(|x|^{-2-m}\right)$.

Proof. The argument is for the most part analogous to that of Theorem 4.3, hence we limit ourselves to pointing out the main remarks. Since $\mathcal{B}_{n}^{n, n-1} \subseteq \mathcal{B}_{1}^{n, n-1}$, the moment condition simplifies to

$$
t \mapsto t^{j} \phi\left(t \omega+\omega^{\perp}\right) \quad \text { is integrable on }(-\infty,+\infty) \text { for all } j=0, \ldots, m .
$$

Pulling back to the sphere by means of Theorem 3.2, this is equivalent to the boundedness of $r^{m+2} \phi\left(r \omega+\omega^{\perp}\right)$ (because the function pulled back is $\mathcal{C}^{\infty}$ on $\mathbf{S}^{n}$ ). But this is clearly equivalent to the boundedness of $R_{0}^{*} \phi(x)|x|^{m+2}$.

Theorem 4.5 yields immediately the following noteworthy generalization of [So, Theorem 8.1] (here $\Lambda=(-\Delta)^{1 / 2}$ ).

Theorem 4.6. For $k=n-1$, let $\phi \in \mathcal{B}_{n}^{n, n-1}$ and assume that $\phi$ satisfies the moment conditions of degree $j=0, \ldots, n-2$. Then

$$
2^{-n} \pi^{1-n} R_{0} \Lambda R_{0}^{*} \phi=\phi .
$$

Let us finally point out that the unusual differentiability conditions appearing in the definitions of the function spaces $\mathcal{A}_{k+1}^{n}, \mathcal{B}_{1}^{n, k}, \mathcal{A}_{1}^{n}, \mathcal{B}_{n}^{n, n-1}$ are automatically satisfied if we restrict our attention to the Schwartz spaces of functions of fast decay. 


\section{REFERENCES}

[BC1] C. A. Berenstein, E. Casadio Tarabusi, Inversion formulas for the $k$-dimensional Radon transform in real hyperbolic spaces, Duke Math. J. 62 (1991), 613-631. MR 93b:53056

[BC2] Range of the $k$-dimensional Radon transform in real hyperbolic spaces, Forum Math. 5 (1993), 603-616. MR 94k:53087

[Gr] E. L. Grinberg, Spherical harmonics and integral geometry on projective spaces, Trans. Amer. Math. Soc. 279 (1983), 187-203. MR 84m:53071

[Gu] V. Guillemin, The Radon transform on Zoll surfaces, Adv. Math. 22 (1976), 85- 119. MR 54:14009

[Hg1] S. Helgason, Groups and geometric analysis: integral geometry, invariant differential operators, and spherical functions, Pure and Appl. Math., vol. 113, Academic Press, Orlando, 1984. MR 86c:22017

[Hg2] - The totally-geodesic Radon transform on constant curvature spaces, Contemp. Math. 113 (1990), 141-149. MR 92j:53036

[Ht] A. Hertle, On the range of the Radon transform and its dual, Math. Ann. 267 (1984), 91-99. MR 86e:44004b

[Ka] T. Kakehi, Range characterization of Radon transforms on $\mathbf{S}^{n}$ and $\mathbf{P}^{n} \mathbf{R}$, J. Math. Kyoto Univ. 33 (1993), 315-328. MR 94g:58221

[Ku1] Á. Kurusa, The Radon transform on hyperbolic space, Geom. Dedicata 40 (1991), 325-339. MR 92k:53130

[Ku2] - The Radon transform on half sphere, Acta Sci. Math. (Szeged) 58 (1993), 143-158. MR 95d:44005

[Ku3] - The invertibility of the Radon transform on abstract rotational manifolds of real type, Math. Scand. 70 (1992), 112-126. MR 93g:44009

[Ku4] - Support theorems for totally geodesic Radon transforms on constant curvature spaces, Proc. Amer. Math. Soc. 122 (1994), 429-435. MR 95a:53111

[MS] W. R. Madych, D. C. Solmon, A range theorem for the Radon transform, Proc. Amer. Math. Soc. 104 (1988), 79-85. MR 90i:44003

[So] D. C. Solmon, Asymptotic formulas for the dual Radon transform and applications, Math. Z. 195 (1987), 321-343. MR 88i:44006

[St] R. S. Strichartz, $L^{p}$ estimates for Radon transforms in Euclidean and non-Euclidean spaces, Duke Math. J. 48 (1981), 699-727. MR 86k:43008

Institute for Systems Research, University of Maryland, College Park, Maryland 20742

E-mail address: carlos@src.umd.edu

Dipartimento di Matematica "G. Castelnuovo", Università di Roma "La Sapienza", Piazzale A. Moro 2, 00185 Roma, Italy

E-mail address: casadio@alpha.science.unitn.it

Bolyai Institute, Aradi vértanúk tere 1., 6720 Szeged, Hungary

E-mail address: kurusa@math.u-szeged.hu 